\title{
Encapsulation of Alendronate in Chitosan based Polymeric Nanoparticles for Effective Management of Osteoporosis - Development to Release Kinetic Study
}

\author{
Sandhya Pathak*, Satyendra Kumar Tripathi, Chandni Pachouri and Archna Pandey \\ Department of Chemistry, Dr. H. S. Gour Central University, Sagar (M.P.), India
}

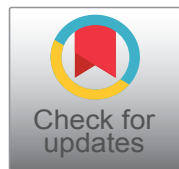

*Corresponding authors: Sandhya Pathak, Department of Chemistry, Dr. H. S. Gour Central University, Sagar (M.P.), 470003, India

\begin{abstract}
Osteoporosis means "Porous bone" is a disease characterized by progressive bone thinning. The deterioration of bone tissue can lead to bone fragility and fracture, especially of the hip, spine, shoulder and wrist. Osteoporosis is characterized by decreasing bone mineral density (BMD). Bisphosphonates are the most commonly prescribed drugs for the treatment of osteoporosis in the US and many other countries including India. Alendronate (Aln) is a widely used anti-osteoporosis drug, exhibits strong inhibitory effect on bone resorption performed by osteoclast cells. Alendronate-sodium is a BCS class III bisphosphonate, used in the treatment of osteoporosis, acts as a potent, specific inhibitor of osteoclast-mediated bone resorption. Alendronate was the first bisphosphonate to be approved for osteoporosis in the US in 1995.

The objective of the present study is to develop, optimize, and evaluate Alendronate-loaded chitosan nanoparticles (NPs) for the treatment of osteoporosis. NPs were prepared by the lonic gelation method and optimized for various parameters. The prepared nanoparticles were characterized using particle size analyser (DLS), transmission electron microscopy (TEM), scanning electron microscopy (SEM) and fourier-transform infrared spectroscopy (FTIR).

Formulated NPs were obtained in the average size ranging from $60 \mathrm{~nm}$ to $220 \mathrm{~nm}$ in TEM, SEM and DLS studies. The release profile was depended on the dissolution medium. The proposed nanoparticles offer an interesting alternative for alendronate delivery via the oral route. Our results indicated that alendronate-loaded chitosan nanoparticles provide an effective medication for the treatment of osteoporosis.
\end{abstract}

\section{Keywords}

Bone mineral density (BMD), Drug loaded nanoparticles, Ion gelation, Osteoporosis, Release kinetics

\section{Introduction}

Osteoporosis is the second most common bone disease with low bone mineral density (BMD) and structural deterioration of bone. In this disease bones become more fragile and porous, it results the reduced bone strength and increased fracture risk. The most effected bones are the hip, spine, shoulder and wrist bones [1]. Decreasing bone density is the main cause of Osteoporosis. The hip and spinal fractures are the most dangerous aspect of osteoporosis. It is commonly seen in old age but women have the high risk after menopause called postmenopausal osteoporosis (PMO). It causes Stooped posture, loss of height and chronic pain with resultant reduction in mobility [2].

There are many risk factors of osteoporosis on the basis of age group and different backgrounds. But these factors may be reason of osteoporosis. Some factors can be controlled and some cannot be controlled are following summarized in Table 1 mentioned by $\mathrm{S}$. Misner, et al. [3,4].

Bisphosphonates are the most commonly used drugs for the treatment of osteoporosis; the efficacy of this drug class for reducing the risks of osteoporosis has been well established in large clinical trials. The strong affinity of bisphosphonates to hydroxyapatite, their relative low toxicity, and their regulatory approval make them agents of choice for bone targeting [3]. Bisphosphonates are used to treat osteoporosis in the US and many other countries including India. Kinetic Study. Int J Med Nano Res 9:036. doi.org/10.23937/2378-3664.1410036 
Table 1: Risk factors of osteoporosis.

\begin{tabular}{|l|l|l|}
\hline S. No. & Controllable Factors & Uncontrollable Factors \\
\hline 1. & Rich diet in calcium or vitamin D & Being older \\
\hline 2. & Proper fruit and vegetable consumption & Female \\
\hline 3. & Balanced diet intake & Postmenopausal \\
\hline 4. & Improvement in everyday lifestyle & Family history \\
\hline 5. & Alcohol consumption & Having small, thin body structure \\
\hline 6. & Smoking & Genetics \\
\hline 7. & Weight loss & Hormonal levels \\
\hline 8. & Medications & Environment with low sunlight \\
\hline
\end{tabular}

Alendronate was the first FDA approved bisphosphonate drug for treatment of osteoporosis in the US in 1995. The osteoclast cells are responsible for bone resorption and caused osteoporosis, so the bisphophonate drugs target to these cells and inhibits the activity of bone resorption. Alendronate is the most potent and recommended drug for the treatment of osteoporosis in medical trials [5]. Alendronate is highly efficient but presents low absorption after oral administration, due to high water solubility fast release occurs. Low systemic bioavailability and burst release of the drug inside the body are main challenges in Alendronate drug delivery systems. Drug nanocarriers help in reducing toxicity, improving solubility and bioavailability, enhancing release and provide better formulation opportunities for drugs $[6,7]$.

Nanotechnology-based drug delivery systems, i.e. polymeric nanoparticles (NPs), solid lipid NPs, liposomes, nanoemulsions, nanosuspension, and micelles etc. have been proved a promising approach for controlled, sustained, and targeted drug delivery in treatment of many diseases. Chitosan (CS) exhibits excellent biocompatibility and biodegradability, and has been extensively used as a carrier for drug delivery systems [8-10].

There are a number of drugs whose clinical development failed due to low bioavailability and other poor biopharmaceutical properties, so presently research work is focused on resolving these problems. The major task in the development of these drugs is the improvement in solubility, thereby enhancing oral bioavailability. The most frequently applied nanotechnology-based strategy in the development of drug delivery systems is polymeric nanoparticles (NPs) based formulations, which present a significant approach for enhancing solubility and oral bioavailability of drugs [11-13].

Chitosan is an important polymeric carrier for many drugs because of its specific properties such as polycationic nature, biodegradability, biocompatibility and non-toxic nature. Chitosan is a natural polysaccharide derived by the process of deacetylation of chitin. Ion gelation is the commonly used method for the formulation of CS NPs. The electrostatic interaction between the amine group of chitosan and a negatively charged group of polyanion such as sodium tripolyphosphate (TPP) is the basic mechanism of this method. In many previous studies, it is reported that different drugs loaded CS nanoparticulate formulations are stable, permeable and therapeutically active $[14,15]$.

In the present study the authors have developed Alendronate loaded CS NPs for better bioavailability and acceptability to the biological systems. Drug delivery systems are designed for promoting the therapeutic effect of a drug and minimizing its toxic side effects, which is achieved by different process variables. The NPs were prepared by lonotropic gelation method and were characterized for average particle size, surface charge, size distribution, drug entrapment efficiency and in vitro drug release. The effect of CS concentration, TPP concentration and stirring speed on the drug entrapment efficiency and particle size was evaluated. Drug delivery systems are designed for promoting the therapeutic effect of Alendronate drug and minimizing its toxic side effects, which is achieved by different process variables. Different mathematical models were used for drug release kinetics study of formulated drug loaded NP [16-19].

\section{Materials and Methods}

\section{Materials}

The drug Alendronate-sodium (M.W. of $249.09 \mathrm{~g} /$ $\mathrm{moL}$ ) was procured from Sigma Aldrich. (Mumbai, India). CS with medium molecular weight (M.W. $=750,000$ Da) was purchased from Himedia (India). Dialysis membrane (Mol. wt. cut-off: 12,000 Dalton, flat with 25 $\mathrm{mm}$, diameter of $16 \mathrm{~mm}$ ) was purchased from Himedia (India). High purity water was used for all experiments, prepared by using (Millipore). All other chemicals and reagents were of analytical grade.

\section{Preparation of Alendronate loaded CS-NPs}

Alendronate loaded CS NPs were prepared by the ionic gelation method [20-24]. In this method, CS-NPs were obtained upon the addition of aqueous sodiumtripolyphosphate (TPP) solution to acidic CS solution stirred at room temperature. NPs formation was the result of ionic interaction between positively charged 
amino groups of CS and negatively charged TPP. In this method, CS was dissolved in $(2 \% \mathrm{w} / \mathrm{v})$ acetic acid solution and stirred to complete dissolution. This acidic chitosan solution was filtered with $0.2 \mu$ filter paper and $\mathrm{pH}$ was adjusted up to $4.8-5.0$ by adding $0.1 \mathrm{M} \mathrm{NaOH}$ solution. A fix amount of drug $(20 \mathrm{mg})$ was added in CS solution after adjusting the $\mathrm{pH}$ and Pluronic F-68 was added as surfactant The CS-NPs were prepared by the drop wise addition of TPP solution to chitosan solution at room temperature on magnetic stirring for 3-4 hours. The process variables such as CS concentration, TPP concentration, stirring speed and $\mathrm{pH}$ for nanoformulations were optimized. The prepared NPs suspension was analysed by transmission electron microscopy (TEM) and Dynamic light scattering (DLS) for particle size. The optimized NPs suspension was centrifuged at $15000 \mathrm{rpm}$ for $30 \mathrm{~min}$ using cooling centrifuge $\left(\mathrm{C}_{24}\right.$, Remi Centrifuge, and Mumbai (India). The pellets were freeze-dried and stored at $5 \pm 3{ }^{\circ} \mathrm{C}$. The weights of freeze-dried nanoparticles were also measured. The entrapment efficiency (\%) was analysed by UV spectrometer for the supernatant. Centrifuged NPs were lyophilized by using lyophilizer (LABCONCO, GNCIIM) for $36 \mathrm{~h}$.

\section{Entrapment efficiency (EE)\% of Alendronate loaded CS-NPs}

The Entrapment efficiency (EE) of Alendronate loaded CS NPs was determined by the indirect method. The nanoparticles were centrifuged at $15,000 \mathrm{rpm}$ for $30 \mathrm{~min}$ and the pellet of NPs was collected and the supernatant was separated. The amount of unentrapped drug in the supernatant was determined by using the method developed by Ostovic, et al. and used by (Cohen-Sela, et al. [25,26]. EE\% was determined at $240 \mathrm{~nm}$ wavelength following the addition of copper (II) reagent ( $5 \mathrm{mM}$ copper sulfate in $1.5 \times 10^{-3} \mathrm{M} \mathrm{HNO}_{3}$ ) by complexation between Alendronate and copper ions. Encapsulated drug amount was obtained by using UVVisible spectrophotometer (UV-1800 Shimadzu, Japan)) after proper dilution. The percentage entrapment efficiency (\% EE) was calculated by using the following formulae:-

Entrapment Efficiency $(\%)=\frac{\text { weight of drug in nanoparticles }}{\text { weight of drug fed initially }} \times 100$

\section{Characterization}

\section{Particle Size, PDI, and zeta potential}

Particle size, Poly dispersive index (PDI) and zeta potential (ZP) of formulated Alendronate loaded CS NPs were determined through dynamic light scattering analysis (DLS) with Malvern Zetasizer Nano S (Malvern, UK).

\section{Surface morphology}

Surface morphology of the best formulation was carried out using scanning electron microscope
(SEM) using (Nanosem, Quantum 200E Instrument). Formulated NPs were also confirmed using Transmission Electron Microscope (TEM) for surface morphology. The prepared sample was examined by TEM (Morgagni 268D TEM, Boston, MA).

\section{Fourier transform infrared (FTIR) studies}

The interaction between drug and polymer was identified from the Fourier transform-infrared, Attenuated total reflection FTIR (ATR-FTIR, Bruker Tensor-37) studies. The FTIR spectrum of pure drug Alendronate, polymer (chitosan), and Alendronate loaded CS NPs were obtained. The samples were prepared by grinding with anhydrous $\mathrm{KBr}$ powder and compressed into pellets The FTIR spectra of drug and drug-loaded NPs were measured over the range of $4000-400 \mathrm{~cm}^{-1}$.

\section{Drug release kinetic studies}

The drug release of NPs was studied using dialysis bag method $[27,28]$. The membrane with a pore size of $2.4 \mathrm{~nm}$ and molecular weight cut-off between 12,000 and 14,000 in phosphate buffer saline (PBS) pH 6.8 at 37 $\pm 2{ }^{\circ} \mathrm{C}$ was used. The drug-loaded NPs were placed into a dialysis membrane, tied at both the ends and placed in a beaker containing $100 \mathrm{~mL}$ of diffusion medium (PBS $\mathrm{pH}$ 6.8). Temperature and speed were maintained at 37 $\pm 2{ }^{\circ} \mathrm{C}$ and $100 \mathrm{rpm}$, respectively, using magnetic stirrer. Aliquot samples were withdrawn at predetermined time intervals, and the same volume was replaced with fresh buffer to maintain the sink condition. The amount of drug released was analyzed spectrophotometrically at $\mathbf{2 4 0} \mathbf{~ n m}$ (complex formation method) for Alendronate drug. Cumulative percentage release was calculated from the amount of drug release. The release kinetics were determined by some mathematical kinetic equations such as zero order, first order, Higuchi's model and Korsmeyer-Peppas model. Values of $R^{2}$ and $K$ were calculated from the linear curve obtained by regression analysis of the plots.

\section{Results and Discussion}

\section{Experimental design}

In the current study, Alendronate loaded CS NPs were prepared by lonic gelation method using polymer (Chitosan), a cross-linking agent sodium tri polyphosphate (TPP) and surfactant (Pluronic F-68). Different formulations were prepared to optimize the process variablesi.e.CSconcentration, TPP concentration and $\mathrm{pH}$ etc. The experimental results from analysis of above formulations, process variance are found to be significant and the optimized formulation is obtained. The results of average particle size and analysis reveal that changing the concentration of CS from $0.5 \mathrm{mg} / \mathrm{ml}$ to $2 \mathrm{mg} / \mathrm{ml}$ have shown slight increase in the particle size, due to the increasing viscosity of chitosan solution. The most significant change in the particle size was 
observed due to increasing concentration of TPP, it was increased the cross-linking density of polymer and drug - polymer electrostatic interaction.

The optimized formulation of Alendronate loaded CS NPs was selected on the basis of the minimum value of particle size and the maximum value of entrapment efficiency. The Optimized formulation has shown average particle size of $\mathbf{2 1 4 . 6} \mathbf{n m}$ and maximum drug entrapment $\mathbf{6 6 . 8 2} \%$. These results showed the best condition for preparing the optimized formulation of Alendronate loaded CS NPs. The above optimized formulation was considered for further studies i.e. characterization and in-vitro drug release kinetic studies.

\section{Characterization}

Fourier transform infra red (FTIR): The FTIR spectra of pure drug Alendronate, polymer Chitosan and drug loaded NPs were obtained by using FTIR to characterize the chemical structure of biopolymer and nanoparticle and obtained spectra are shown in Figure $1 \mathrm{a}$, Figure $1 \mathrm{~b}$ and Figure 1c.
The main characteristic peaks of Alendronatesodium shows at $1186.45 \mathrm{~cm}^{-1}$ and $1063.91 \mathrm{~cm}^{-1}$ (in the region $\left.1200-900 \mathrm{~cm}^{-1}\right)$ correspond to ( $C=0$ stretching), and $(\mathrm{P}=\mathrm{O}$ stretching) respectively. FTIR spectra of Chitosan shows the characteristic peaks at $3370.57 \mathrm{~cm}^{-1}$ ( $\mathrm{OH}$ and $\mathrm{NH}_{2}$ stretching) and $2890.44 \mathrm{~cm}^{-1}$ due to $\mathrm{C}-\mathrm{H}$ stretching. The spectrum of drug loaded NPs shows new characteristic absorption peak at $1230.77 \mathrm{~cm}^{-1}$ for (C-O-C stretching) and $1541.02 \mathrm{~cm}^{-1}$ for (amide II) which indicated the electrostatic interaction between amine groups of chitosan and phosphoric groups of sodium tri poly phosphate (TPP). A new peak at $881.41 \mathrm{~cm}^{-1}$ confirmed the encapsulation of drug Alendronate inside the NPs [29-31].

Nanoparticle size: Alendronate loaded CS NPs were prepared by the ion gelation method. This method was used to prepare the desired NPs and considered to be adequate nanocarriers for the encapsulation of drug due to its easy methodology, cost-effectiveness and non-toxic nature. The prepared Alendronate loaded CS NPs were found in nano range and their spherical (a)

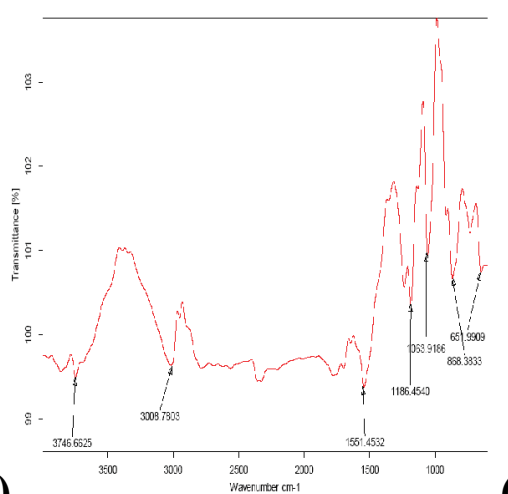

(b)

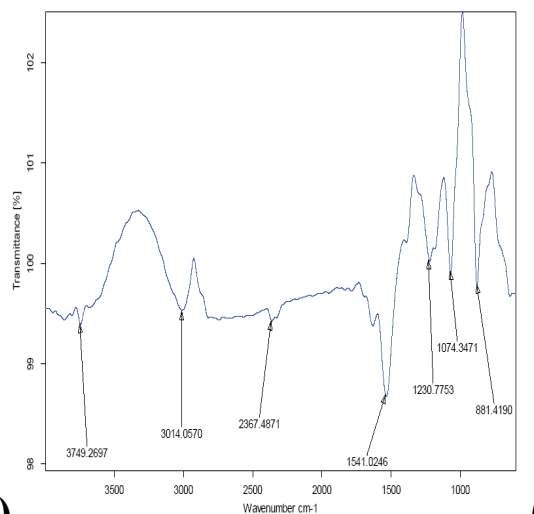

(c)

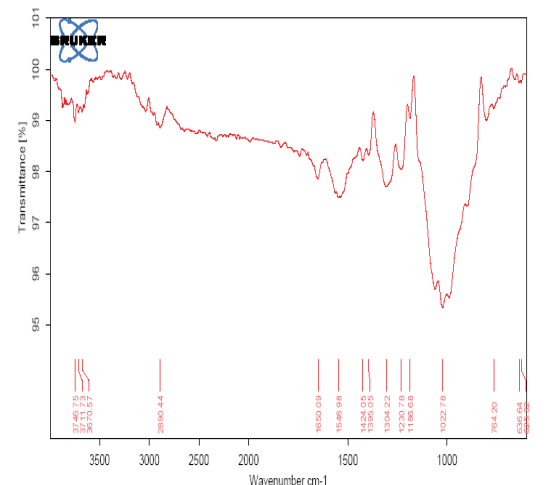

Figure 1: FTIR spectra of (a) Alendronate (API); (b) Alendronate CS NPs; (c) Chitosan.

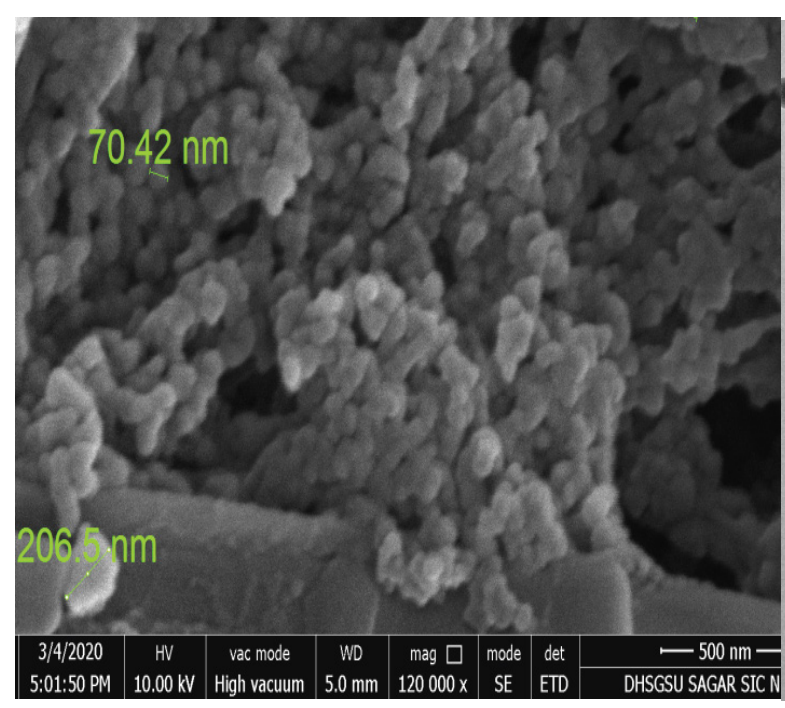

(a)

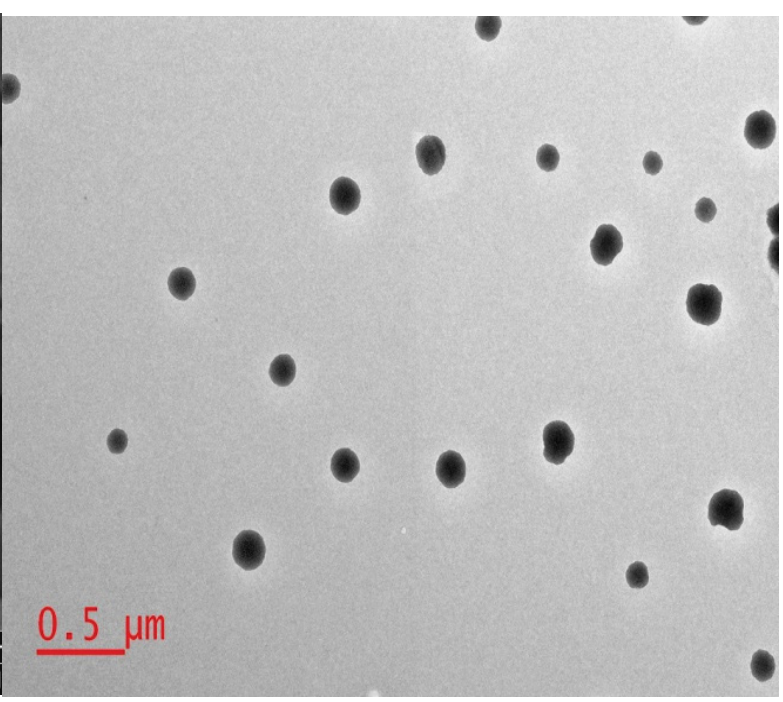

(b)

Figure 2: Images ( $a \& b)$ elaborating SEM and TEM analysis of synthesized Alendronate- CS NPS. 
shapes were confirmed using TEM and SEM in Figure $2 a$ and Figure $2 \mathrm{~b}$. The particle size and Zeta potential were analyzed by Malvern Nano Zetasizer as shown in Figure $3 a$ and Figure $3 b$.

The morphology of nanoparticles was analysed by using to SEM, the scanning Electron Microscopic studies shows the particle size in the range of $200 \mathrm{~nm}$. The particle size of optimized NPs formulation was also characterized with TEM and the result was found to be in the range below $200 \mathrm{~nm}$. TEM image showed completely spherical and symmetrical nanoparticles were formed in the optimized formulation.
Based on the results of particle size and entrapment efficiency, the optimized formulation showed the lesser particle size $(\mathbf{2 1 4 . 6} \mathbf{n m})$ than the other batches with higher entrapment efficiency (66.82\%) and poly dispersive index (PDI) was $\mathbf{0 . 1 5 6}$. The zeta potential of the optimized formulation was found to be $+\mathbf{2 4 . 1} \mathbf{~ m V}$. On the basis of results found in the optimization studies, the best ratio between polymer (CS) and crosslinking agent (TPP), (2:0.5) $\mathrm{mg} / \mathrm{ml}$ was found optimum in which the particle size was found in the range below $200 \mathrm{~nm}$.

Differential scanning calorimetry (DSC): DSC analysis is important to identify possible interactions between the
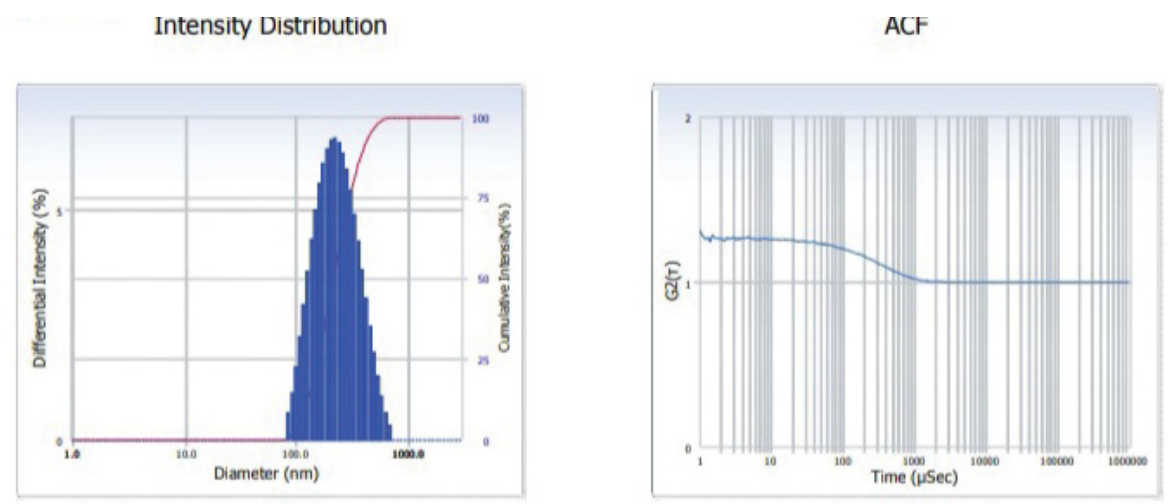

Fig.3 (a)

Zeta Potentlal Distribu:ion

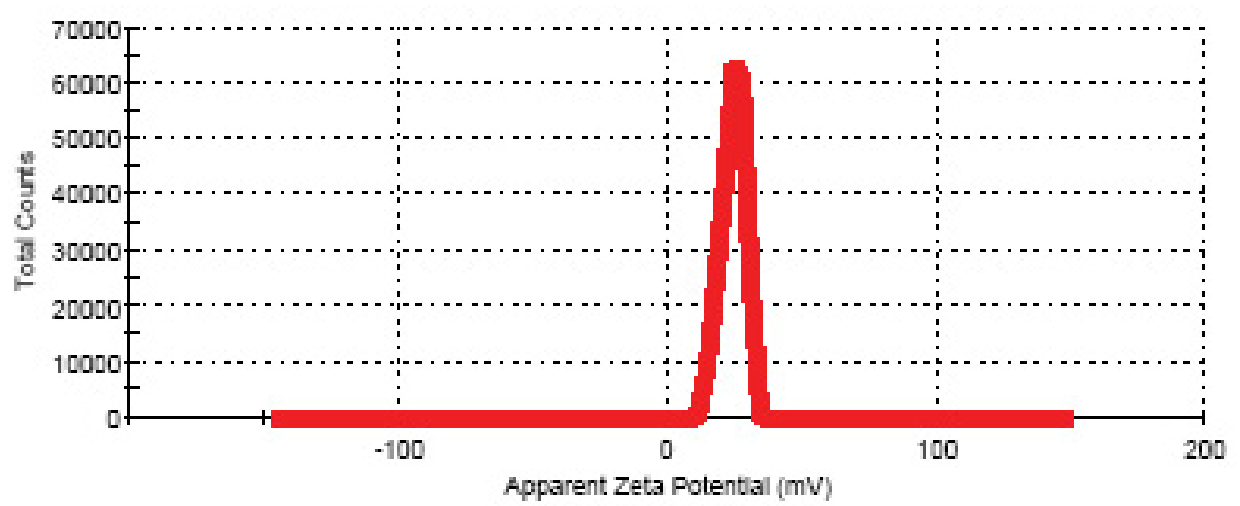

Fig.3(b)
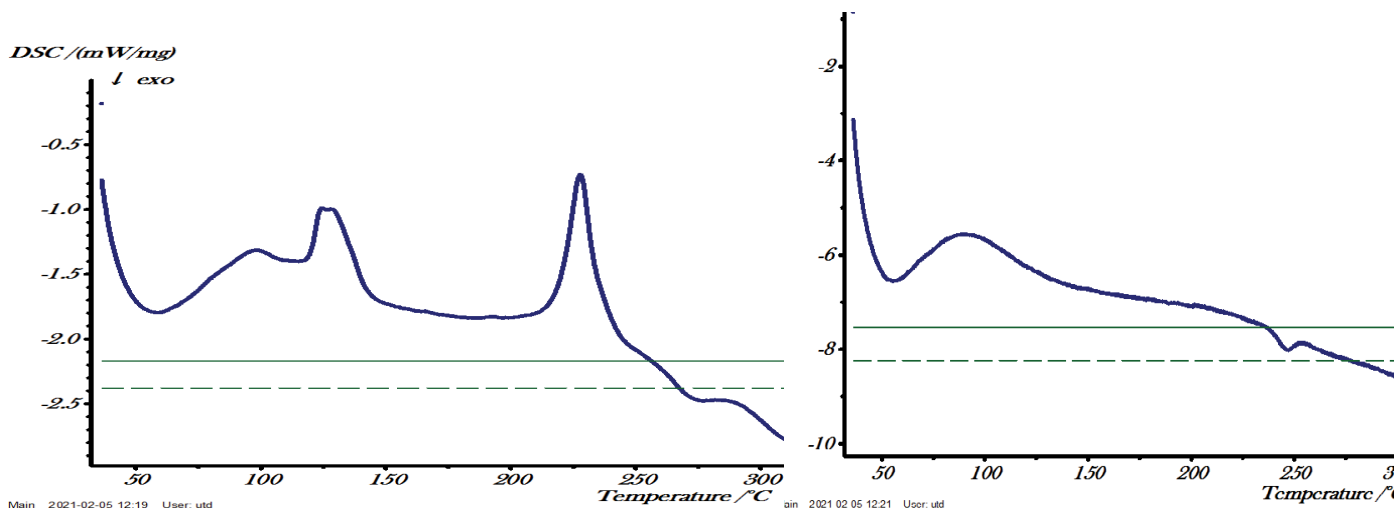

Fig3(c \& d)

Figure 3: (a) Average Particle size of Alendronate- CS NPs; (b) Zeta Potential of Alendronate CS NPs; (c) DSC curve of Alendronate (API); (d) DSC curve of Alendronate.

Images $3(\mathrm{a} \& \mathrm{~b})$ showing zeta sizer analysis of synthesized Alendronate- CS NPs (Mean \pm SD, $n=3$ ), (c \& d) Elaborating the DSC curve of Alendronate (API) and NPs. 
components of nano-formulation i.e. drug compatibility study. The DSC thermograms of pure drug Alendronate and Drug loaded polymeric nanoformulation are shown in Figure $3 c$ and Figure 3d. Thermal analysis of pure drug Alendronate showed a sharp peak at $234{ }^{\circ} \mathrm{C}$, indicating its sharp crystalline nature. This temperature corresponding to its melting point. The melting peak drug was not detected in the NP formulation, indicating that the drug was encapsulated into the NPs and decreased crystallinity indicates the change in solid state structure of chitosan due to cross-linking by TPP. The amorphous form of the drug circulates freely in the systemic circulation. The compatibility study of drug and excipients was identifying by DSC and shows the drug and excipients are compatible with each other and can be used for further development of formulation.

\section{Drug release kinetic study}

In drug release study, ADME process is involved, in which drug molecules migrate from the initial position and available for the biological action in to the systemic circulation. In vitro drug release of formulated Alendronate loaded CS NPs was compared with pure drug solution shown in Figure 4. Results showed that release of pure drug was fast, about $90 \%$ drug release within 8-10 hours, while CS NPs formulation showed the sustained release of drug up to $30 \mathrm{~h}$. Initially, CS NPs formulation showed the burst release ( $45 \%$ drug releases in $10 \mathrm{~h}$ ), followed by sustained releases up to $30 \mathrm{~h}$. Initial fast release may be due to the presence of the adsorbed drug on the surface of CS NPs.
In-vitro drug release studies were carried out using dialysis bag method for different mathematical kinetic models. For kinetic study of formulated drug NPs, the plots were made for Zero order kinetic model (cumulative $\%$ drug release vs. time), First order kinetic model (log of cumulative \% drug remaining vs. time), Higuchi model cumulative (\% drug release vs. square root of time) and Korsmeyer-Peppas model (log cumulative \% drug release vs. log time).

Plots of above mentioned models are shown in Figure 5 and results are summarized in Table 2 . In the above table " $\mathbf{R}$ " is correlation value, " $\mathrm{K}$ " is rate constant and " $n$ " is release exponent.

\section{Different mathematical models}

On the basis of above values of $\left(R^{2}\right)$, the best fit kinetic model with the highest correlation value is Zero order model. It is concluded that in the optimized formulated NPs follow Zero order model kinetics. In the KorsmeyerPeppas model, release exponent value " $n$ " is 0.47 . The magnitude is in the range $(0.45<n<0.89)$ indicates the release mechanism is non-Fickian diffusion.

\section{Conclusion}

Alendronate is the most efficient bisphosphonate for the treatment of Osteoporosis, but there is increasing concern about their long-term safety. Medications with novel mechanisms and novel formulation of drug loaded polymeric NPs can be expected to treat osteoporosis in future. The results of the current study would help us to find a new approach for drug discovery and

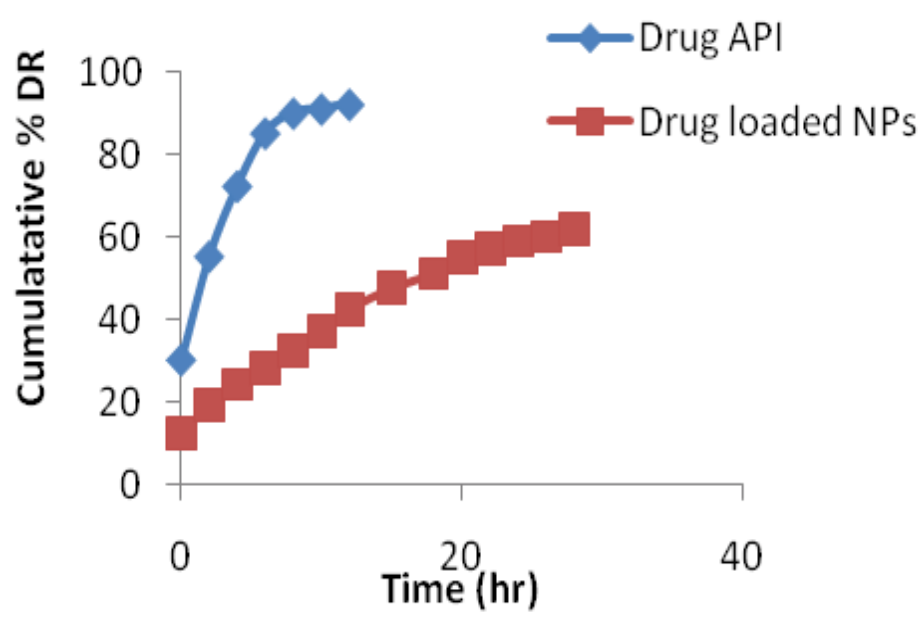

Figure 4: In-vitro drug release profile of Alendronate loaded CS NPs and Pure drug in PBS (pH 6.8).

Table 2: Interpretation of R2 values and rate constants $(K)$ of release kinetics of NPs.

\begin{tabular}{|l|l|l|}
\hline Kinetic Models & Correlation value $\left.\mathbf{( R}^{\mathbf{2}}\right)$ & Release exponent (n) \\
\hline Zero order model & 0.9755 & ---- \\
\hline First order model & 0.8667 & ---- \\
\hline Higuchi model & 0.9728 & ----- \\
\hline Korsmeyer-Peppas model & 0.848 & 0.47 \\
\hline
\end{tabular}




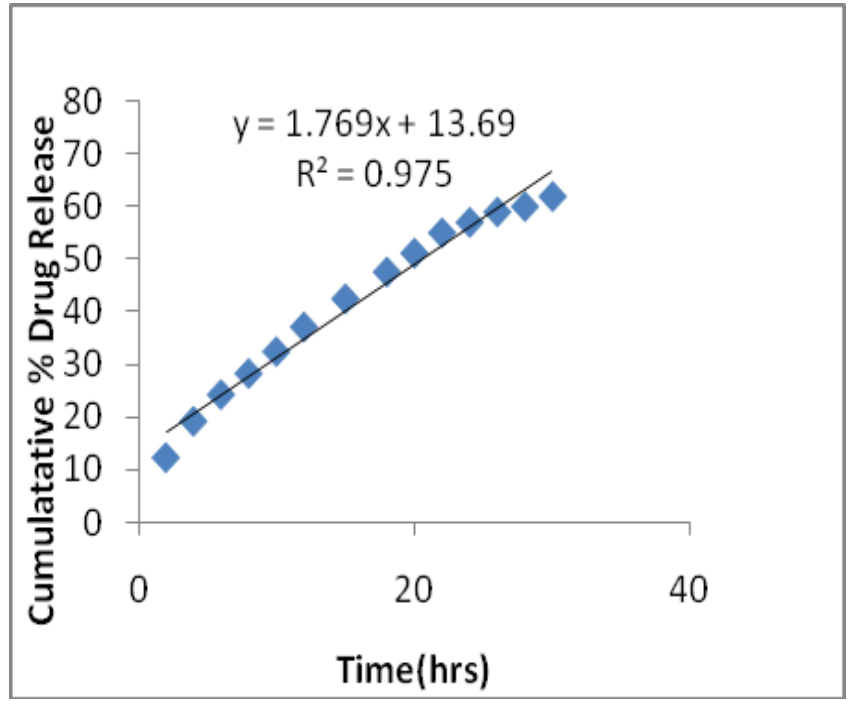

(a)

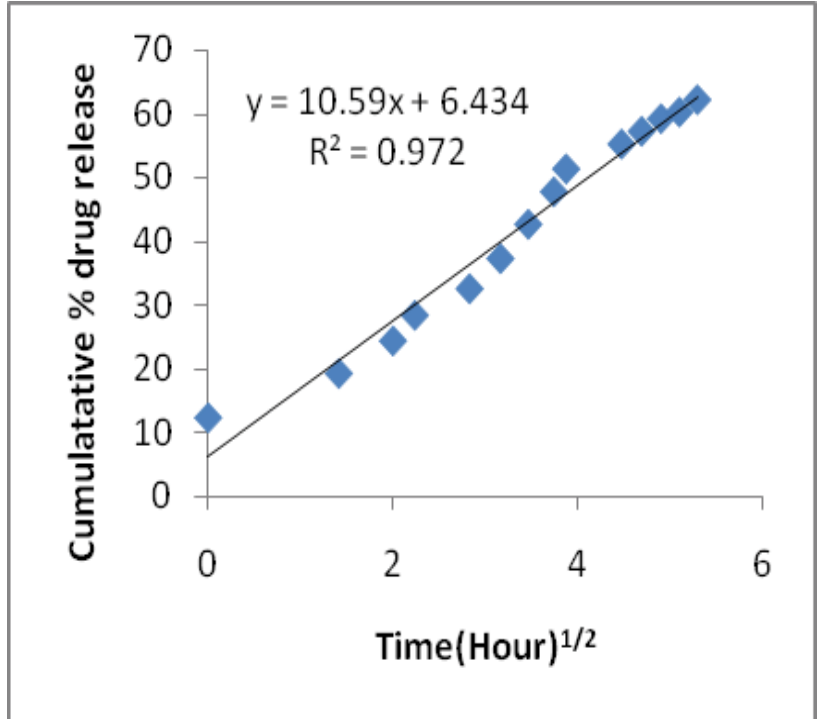

(c)

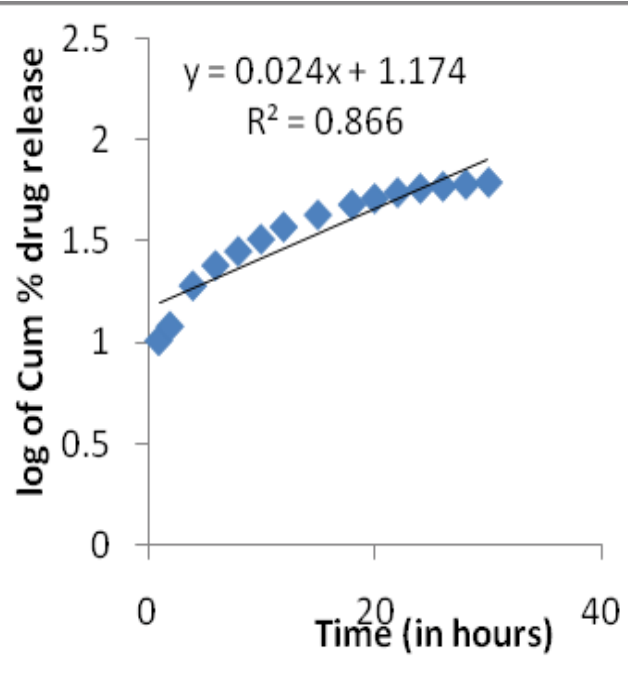

(b)

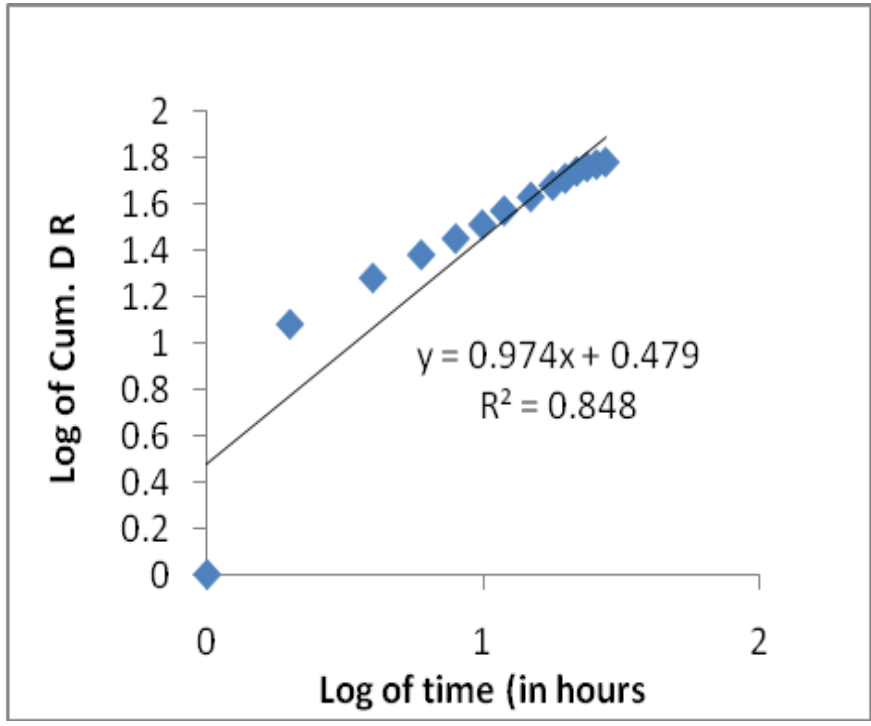

(d)

Figure 5: (a) Zero order plot; (b) First order plot; (c) Higuchi model plot; (d) Korsemeyer - Peppas model.

drug delivery by preparing the anti-osteoporotic drug Alendronate in the Nano-range. Alendronate loaded CS NPs prepared via ionic gelation method presented the significant results of drug release profile and increased the therapeutic efficacy of Alendronate drug for the effective treatment of osteoporosis.

\section{Acknowledgement}

The authors are grateful to Electron Microscope Unit AIIMS, New Delhi and IISER (Bhopal) for DLS studies. We acknowledge Sophisticated Instrumentation Center (SIC), Dr. Harisingh Gour Vishwavidyalaya (A Central University), Sagar for providing us the SEM, TEM, FTIR and DSC facilities.

\section{References}

1. Anna D (2012) Prevention and treatment of osteoporosis in women: An update. Obstet Gynaecol Reprod Med 22: 162-169.
2. Kawalkar AC (2014) A comprehensive review on osteoporosis. Journal of Trauma \& Orthopaedics 9: 3-12.

3. Misner S, Farrell VA (2017) Osteoporosis Revised: AZ9712.

4. Pouresmaeili F, Kamalidehghan B, Kamarehei M, Goh YM (2018) A comprehensive overview on osteoporosis and its risk factors. Ther Clin Risk Manag 14: 2029-2049.

5. Giger EV, Castagner B, Leroux JC (2013) Biomedical applications of bisphosphonates. J Control Release 167: 175-188.

6. Khadilkar AV, Mandlik RM (2015) Epidemiology and treatment of osteoporosis in women: An Indian perspective. Int J Womens Health 7: 841-850.

7. Rumian L, Wolf-Brandstetter C, Rößler S, Reczyńska K, Tiainen $H$, et al. (2020) Sodium alendronate loaded poly (I-lactide-co-glycolide) microparticles immobilized on ceramic scaffolds for local treatment of bone defects. Regen Biomater 7: 293-302.

8. Miladi K, Sfar S, Fessi H, Elaissari A (2013) Drug carriers in osteoporosis: Preparation, drug encapsulation and applications. Int J Pharm 445: 181-195. 
9. Miladi K, Sfar S, Fessi H, Elaissari A (2015) Encapsulation of alendronate sodium by nanoprecipitation and double emulsion: From preparation to in vitro studies. Industrial Crops and Products 72: 24-33.

10. Casettari L, Illum L (2014) Chitosan in nasal delivery systems for therapeutic drugs. J Control Release 190: 189200.

11. De Pinho NAL, Milioli CC, Müller L, Riella HG, Kuhnen NC, et al. (2014) Factorial design as tool in chitosan nanoparticles development by ionic gelation technique. Colloids and Surfaces A: Physicochemical and Engineering Aspects 445: 34-39.

12. Ruttala HB, Ko YT (2015) Liposomal co-delivery of curcumin and albumin/paclitaxel nanoparticle for enhanced synergistic antitumor efficacy. Colloids Surf B Biointerfaces 128: 419-426.

13. Liu YF, Liu R, Li XY, Song Z, Zhao XH (2016) Development of docetaxel and alendronate-loaded chitosanconjugated polylactide-co-glycolide nanoparticles: In vitro characterization in osteosarcoma cells. Trop J Pharm Res 15: 1353

14. Ahmed TA, Aljaeid BM (2016) Preparation, characterization, and potential application of chitosan, chitosan derivatives, and chitosan metal nanoparticles in pharmaceutical drug delivery. Drug Des Devel Ther 10: 483-507.

15. Puramdas E, Singh C, Kumar V, Bhatt TD, Roy S, et al. (2016) Enhanced Solubility and Dissolution Rate of Raloxifene using Cycloencapsulation Technique. J Anal Pharm Res 2: 00032.

16. Pathak S, Tripathi S, Shukla S, Pandey A (2020) Nanotechnology: An emerging field of osteoporosis treatment and kinetic models for drug release studies- $A$ review. SIPN 40.

17. Patel B, Tripathi S, Shroti S, Shukla S, Pandey A (2020) Antimalarial drugs and their nanoparticles - A societal impact. Oxidation Communications 43: 371-393.

18. Tripathi SK, Patel B, Shukla S, Pachouri C, Pathak S, et al. (2021) Donepezil loaded PLGA nanoparticles, from modified nano-precipitation, an advanced drug delivery system to treat alzheimer disease. NCRAPS 1849: 012001.

19. Ramteke KH, Dighe PA, Kharat AR, Patil SV (2014) Mathematical models of drug dissolution: A review. Sch Acad J Pharm 3: 388-396.

20. Calvo P, Remunan-Lopez C, Vila-Jato JL, Alonso MJ (1997) Chitosan and chitosan/ethylene oxide-propylene oxide block copolymer nanoparticles as novel carriers for proteins and vaccines. Pharm Res 14: 1431-1436.
21. Iswandana R, Putri KSS, Dwiputra R, Yanuari T, Sari SP, et al. (2017) Formulation of chitosan tripolyphosphatetetrandrine beads using ionic gelation method: In vitro and in vivo evaluation. Int J App Pharm 9: 109-115.

22. Severino PC, da Silva CF, da Silva MA, Santana MHA, Souto EB (2014) Chitosan cross-linked pentasodium tripolyphosphate micro/nanoparticles produced by ionotropic gelation. Sugar Tech 18: 49-54.

23. Sailaja AK, Amareshwar P, Chakravarty P (2011) Different techniques used for the preparation of nanoparticles using natural polymers and their application. Int J Pharm Pharm Sci 3: $45-50$.

24. Othman N, Masarudin MJ, Kuen CY, Dasuan NA, Abdullah LC, et al. (2018) Synthesis and optimization of chitosan nanoparticles loaded with L-Ascorbic acid and thymoquinone. Nanomaterials (Basel) 8: 920.

25. Cohen-Sela E, Chorny M, Koroukhov N, Danenberg HD, Golomb G (2009) A new double emulsion solvent diffusion technique for encapsulating hydrophilic molecules in PLGA nanoparticles. J Control Release 133: 90-95.

26. Cohen-Sela E, Rosenzweig O, Gao J, Epstein H, Gati I, et al. (2006) Alendronate-loaded nanoparticles deplete monocytes and attenuate restenosis. J Control Release 113: 23-30.

27. Weng J, Tong HHY, Chow SF (2020) In Vitro release study of the polymeric drug nanoparticles: Development and validation of a novel method. Pharmaceutics 12: 732.

28. Zambito Y, Pedreschi E, Colo GD (2012) Is dialysis a reliable method for studying drug release from nanoparticulate systems? A case study. Int J Pharm 434: 28-34.

29. Avadi MR, Sadeghi AMM, Mohammadpour N, Abedin S, Atyabi F, et al. (2010) Preparation and characterization of insulin nanoparticles using chitosan and Arabic gum with ionic gelation method. Nanomedicine 6: 58-63.

30. Hosseini SF, Zandi M, Rezaei M, Farahmandghavi F (2013) Two-step method for encapsulation of oregano essential oil in chitosan nanoparticles: Preparation, characterization and in vitro release study. Carbohydr Polym 95: 50-56.

31. Boanini E, Torricelli P, Gazzano M, Fini M, Bigi A (2012) The effect of alendronate doped calcium phosphates on bone cells activity. Bone 51: 944-952. 